\title{
A Equipe de Saúde Bucal e as práticas de Vigilância em Saúde no território
}

\section{The Oral Health Team and Health Surveillance practices in the territory}

\section{El equipo de salud bucal y las prácticas de vigilancia sanitaria en el território}

Júlya Karolina Gomes ${ }^{1}$ Ana Lúcia Guerra de Aquino Albuquerque ${ }^{2}$ Inês Patrícia Guedes Souto ${ }^{3}$ Márcia Maria Dantas Cabral Melo ${ }^{4}$

RESUMO: Objetivou-se analisar as práticas de vigilância em saúde de equipes de saúde bucal (eSB) da Estratégia Saúde da Família do município de Jaboatão dos Guararapes/PE. Estudo descritivo com amostra representada pelos dentistas (31) das eSB de três regionais de saúde. Para coleta de dados, realizou-se validação do instrumento com as entrevistas aplicadas face a face. As análises foram descritivas. Participaram 27 dentistas. Observou-se práticas de vigilância das eSB no território, contudo menos da metade possui vínculos fortes(famílias, 48,1\%; organizações comunitárias, 18,5\%). Realizam às vezes levantamentos epidemiológicos $(51,9 \%)$ com prioridade para cárie $(51,9 \%)$. As ações promocionais/educativas de abordagem comunitária $(74,1 \%)$ são realizadas com metodologias conservadoras $(71,7 \%)$ prevalecendo o grupo alvo escolar $(74,2 \%)$. Mas existe atuação integrada com demais membros da equipe para planejamento, ações educativas, busca ativa e visitas domiciliares. Conclui-se que as práticas de vigilância em saúde bucal no território ainda são restritas, mas com esforços de ampliação e atuação interprofissional como requerido.

Palavras-chave: Saúde bucal. Atenção básica. Processo de trabalho. Vigilância em Saúde.

1 Cirurgiã-dentista graduada pela Universidade Federal de Pernambuco, UFPE.

2 Mestre em Gestão e Economia da Saúde pela UFPE. Cirurgiã-dentista servidora da Prefeitura de Jaboatão dos Guararapes, atuando na Atenção Básica. Docente do Curso de Especialização em Saúde Pública, pela ESPPE. 3 Especialista em Saúde Pública pelo Ageu Magalhães/FIOCRUZ e em Saúde da Família pela FACISA. Cirurgiãdentista servidora da Prefeitura do Recife na Estratégia de Saúde da Família.

4 Doutora em Saúde Pública pelo Instituto de Pesquisa Aggeu Magalhães, Fiocruz-Pernambuco. Prof ${ }^{a}$ Adjunta de Saúde Coletiva do Departamento de Clínica e Odontologia Preventiva da Universidade Federal de Pernambuco. 
ABSTRACT: The objective of this study was to analyze the health surveillance practices performed by Oral Health Teams (eSB) of the Family Health Strategy (ESF) in the municipality of Jaboatão dos Guararapes/PE. Descriptive study with sample represented by the dentists (31) of the eSB of three regional health. For data collection, the instrument was validated with faceto-face interviews. The analyzes were descriptive. 27 dentists participated. Surveillance practices were observed in the territory, but less than half had strongs bonds (families, 48,1\%; community organizations, $18,5 \%)$. They carry out epidemiological surveys $(51.9 \%)$ with priority for caries (51.9\%). The promotional / educational actions of a community approach (74.1\%)\%) are carried out with conservative methodologies $(71.7 \%)$, with the school target group $(74.2 \%)$ prevailing. But there is integrated action with other team members for planning, educational actions, active search and home visits. It is concluded that the practices of oral health surveillance in the territory are still restricted, but with efforts of expansion and interprofessional action as required.

Keywords: Oral health. Basic Attention. Work process. Health Surveillance.

RESUMEN: Este estudio tuvo como objetivo analizar las prácticas de vigilancia de la salud de los equipos de salud bucal (eSB) de la Estrategia de Salud Familiar de la ciudad de Jaboatão dos Guararapes / PE. Estudio descriptivo con muestra representada por dentistas (31) del eSB de tres regiones sanitarias. Para la recopilación de datos, el instrumento fue validado con entrevistas personales. Los análisis fueron descriptivos. Participaron 27 dentistas. Se observaron prácticas de vigilancia de ESB en el territorio, pero menos de la mitad tenían lazos fuertes (familias, 48.1\%; organizaciones comunitarias, $18.5 \%)$. A veces realizan encuestas epidemiológicas $(51,9 \%)$ con prioridad para la caries $(51,9 \%)$. Las acciones de promoción / educación basadas en la comunidad (74.1\%) se llevan a cabo con metodologías conservadoras (71.7\%), prevaleciendo el grupo escolar objetivo (74.2\%). Pero existe una acción integrada con otros miembros del equipo para la planificación, acciones educativas, búsqueda activa y visitas a domicilio. Se concluye que las prácticas de vigilancia de la salud oral en el territorio aún están restringidas, pero con esfuerzos de expansión y desempeño interprofesional según se requiera.

Palabras clave: Salud Oral. Atención primaria Proceso de trabajo. Vigilancia de la salud.

\section{INTRODUÇÃO}

Desde a criação do Sistema Único de Saúde (SUS) em 1988, a saúde pública brasileira tem como objetivo a substituição do modelo de atenção à saúde médico assistencial-privatista até então hegemônico, para um modelo assistencial sanitarista. ${ }^{1}$ Esses dois principais modelos convivem, ora em caráter complementar ora de maneira contraditória, caracterizando um sistema de saúde pautado por uma prática fragmentada e ofertada de maneira desigual e descontextualizada. Diante disso, tornou-se consenso que o sistema de saúde brasileiro necessita rever a lógica tradicional que ainda o rege, buscando condições para que, de forma permanente, consiga uma aproximação dos indivíduos, tornando-se mais humanizado e resolutivo. A vigilância da saúde encontra-se entre as principais propostas de mudança na organização das práticas de saúde. ${ }^{2}$

Tempus, actas de saúde colet, Brasília, 14(1), 45-63, mar, 2020. Epub Mai/2020 ISSN 1982-8829 
No contexto atual brasileiro, a vigilância à saúde é entendida como um componente estrutural da maior importância na organização e gestão das práticas do SUS, por fornecer métodos, tecnologias e conhecimentos para atender as necessidades de saúde de um dado território populacional. ${ }^{3} \mathrm{~A}$ Vigilância à Saúde está centrada nos pilares: território, participação popular e intersetorialidade e incorpora o conceito de processo de trabalho em saúde. Tem sido compreendida de três formas: (1) como análise e monitoramento de situações de saúde; (2) como integração institucional entre atividades de vigilância epidemiológica e sanitária; e (3) como elemento que pressupõe a organização tecnológica do trabalho de redefinição das práticas sanitárias. ${ }^{3,4}$

O conceito de Vigilância em Saúde (VS) é novo no país e é frequentemente confundida com duas áreas específicas: a vigilância epidemiológica e/ou vigilância sanitária, devido à própria historicidade da saúde coletiva no Brasil cuja principais ações de vigilância no passado baseavam-se no controle de doenças transmissíveis e nas ações de saneamento dos centros urbanos econômicos. No entanto, o atual cenário epidemiológico brasileiro e mundial apresenta uma tripla carga de problemas de saúde na população (doenças transmissíveis, não transmissíveis e causas externas) exigindo, desta forma, uma ampliação da capacidade de respostas da saúde pública frente a estas problemáticas. ${ }^{5}$

O novo desafio da saúde pública é a atuação interdisciplinar e interprofissional como requisito básico para a integralidade das ações articuladas e integradas atendendo as especificidades e características de cada território. A execução das ações de VS são também atribuições dos profissionais da atenção básica. As equipes de VS devem fornecer orientação técnica permanente a esses profissionais e trabalhar de forma integrada. ${ }^{2,4,6}$

No Brasil, a rede de Atenção Primária à Saúde (APS) se constitui num lócus privilegiado para as práticas de vigilância à saúde e saúde bucal sendo parte estruturante da VS..${ }^{1,7}$ A APS atua como porta de entrada do usuário, sendo o primeiro contato deste com o sistema de saúde e assim contempla os elementos que dão subsídios para execução da VS. Tornando-se a integração entre a VS e a APS uma condição obrigatória para a construção da integralidade na atenção e para o alcance dos resultados em saúde, com desenvolvimento de um processo de trabalho condizente com a realidade local, que preserve as especificidades dos setores e compartilhe suas tecnologias. ${ }^{4}$

Para Pinto, Pereira e Limongi ${ }^{1}$ na APS a Estratégia Saúde da Família (ESF) tem como sustentação os princípios da VS, garantindo o acompanhamento do paciente desde seu primeiro contato, até o desenrolar final, conhecendo mais a fundo seu território e seu círculo social.

Isto significa que as equipes de saúde devem agir em comunicação de acordo com as diferentes fases ou dimensões do processo saúde-doença, desde os agravos a situações de exposição, às necessidades sociais de saúde. No entanto, de acordo com Goldstein ${ }^{8}$, as informações de vigilância e atenção à saúde produzidas no nível local contribuem para as análises em macroescalas, mas são pouco analisadas e utilizadas no nível local, e os indicadores gerados por esses serviços acabam por 
auxiliarem pouco no processo de trabalho da equipe da ESF.

No tocante às ações de saúde bucal, dentro do SUS estas vêm num processo de ampliação com a inclusão da equipe de saúde bucal (eSB) na ESF e a implementação da Política Nacional de Saúde Bucal (PNSB), a partir de 2004, que propõe um modelo centrado nas necessidades de saúde da população, por meio da reorganização do processo de trabalho e da qualificação dos serviços oferecidos pelas eSB. ${ }^{9}$

Discute-se que a implementação da Vigilância à Saúde Bucal (VSB) integrada estrategicamente ao Sistema Nacional de Vigilância em Saúde (SNVS) é um elemento essencial para sustentar a PNSB. Sendo considerado o Projeto SB Brasil, por exemplo, uma estratégia central de vigilância através da produção de dados primários de saúde bucal, contribuindo para a construção de modelos de atenção de base epidemiológica. ${ }^{10}$

Contudo, as informações sobre saúde bucal contidas nos sistemas de informação se consolidaram basicamente em função daquelas sobre produção ambulatorial e de poucos indicadores propostos pelo pacto de atenção básica. ${ }^{10,11}$ Fato que dificulta a efetivação do modelo de atenção em saúde bucal pautado na vigilância das ações de saúde bucal a nível local.

Ademais, estudos recentes informam uma série de problemas enfrentados pelas eSB para atuarem na perspectiva da integralidade. Sá, Kuhnen, Santos, Arruda e Toassi ${ }^{12}$ verificaram que as práticas odontológicas no SUS se apresentam de forma desarticulada ao processo de organização dos demais serviços de saúde da ESF, devido à ausência de planejamento em saúde. Machado, Souza e Noro ${ }^{11}$ discutem que, independente do nível de atenção, a análise e o uso da informação ainda são precários e existem poucos indicadores para o monitoramento e avaliação das ações locais de VSB.

Diante disso, o objetivo deste estudo foi realizar uma análise das práticas de VS desenvolvidas pelas eSB da ESF do município de Jaboatão dos Guararapes/PE. A intenção é subsidiar com evidências o planejamento local em saúde.

\section{METODOLOGIA}

Trata-se de um estudo descritivo com abordagem quantitativa para caracterizar as práticas de vigilância em saúde bucal das eSB.

O estudo foi realizado no município de Jaboatão dos Guararapes/PE, que possui a rede de atenção à saúde distribuída por sete regionais de saúde e dispõe de 83 unidades de saúde da família (USF), 73 eSB, 12 unidades básicas de saúde (UBS) sendo oito delas com eSB e seis USF em construção com oito eSB. ${ }^{13}$

A população do estudo foi constituída pelas eSB das USF das regionais I, II e III do referido 
Município. A amostra foi representada pelo membro Cirurgião-Dentista (CD) das eSB, o que correspondeu a $31 \mathrm{CD}$. Foram incluídos no estudo todos os profissionais que estavam em exercício de suas funções a mais de seis meses nas USF das Regionais selecionadas para o estudo e aceitaram participar da pesquisa, após a assinatura do Termo de Consentimento Livre e Esclarecido. Foram excluídos os profissionais das eSB que estavam de licença ou férias, ou que apresentavam deficiência auditiva.

Para a coleta de dados foi elaborado um questionário do tipo estruturado e auto-aplicável. A elaboração do formulário de entrevista (questionário) foi precedida por revisão bibliográfica, em consonância com o referencial teórico adotado pelo estudo e, posteriormente, houve a validação de face do instrumento por dois especialistas com expertise relacionada à problemática, segundo os critérios recomendados para garantir o controle de qualidade dos dados.

O instrumento de entrevista foi organizado em quatro blocos. O primeiro bloco incluiu variáveis para caracterizar o perfil profissional, como: idade; sexo; tempo de formação; tempo de atuação na ESF e forma de ingresso (concurso, seleção simplificada, outro). O segundo bloco foi composto por variáveis relacionadas ao vínculo da eSB com a população adstrita à USF. O terceiro bloco foi composto por variáveis referentes à atuação da eSB nas práticas de VSB. O quarto e último bloco foi composto por variáveis sobre a integração da eSB com a equipe de Saúde da Família (eSF) no planejamento das ações de saúde.

A análise dos dados da amostra estudada deu-se por meio de cálculos de estatística descritiva. Foi calculada a distribuição de frequência absoluta e relativa, para cada uma das variáveis e/ou questões estudadas. A digitação dos dados e os cálculos estatísticos foram realizados em planilhas do programa Microsoft Excel, versão 2007.

O projeto de pesquisa foi aprovado pelo Comitê de Ética em Pesquisas do Centro de Ciências da Saúde da UFPE, CAAE: 99704818.9.0000.5208 Número do Parecer: 2.965.569.

\section{RESULTADOS}

Da amostra total de $31 \mathrm{CD}$ pertencentes às regionais I, II e III da APS do Jaboatão dos Guararapes planejada para participar deste estudo, quatro não foram entrevistados por não estarem presentes no momento da coleta de dados, significando uma perda de $12,9 \%$.

Os dados relativos à caracterização demográfica e profissional dos entrevistados estão expostos na Tabela 1. Referente à idade, as informações revelaram que 66,7\% ( $\mathrm{n}=18)$ encontravam-se na faixa etária de 28 a 38 anos e 14,8\% (n=4) na faixa de 41 a 55 anos. Relativo ao sexo, a maioria era do sexo feminino correspondendo a 70,4\% $(\mathrm{n}=19)$ e apenas $11,1 \%(\mathrm{n}=3)$ era do sexo masculino.

Sobre a formação, 22,2\% $(\mathrm{n}=6)$ dos entrevistados possuíam de 7 a 9 anos de formados, 18,5\% $(\mathrm{n}=5)$ afirmaram ter de 4 a 6 anos de formados e 18,5\% $(\mathrm{n}=5)$ de 10 a 14 anos. Grande parte (40,7\%; 
$\mathrm{n}=11)$ informou atuar na ESF de 1 a 3 anos e 29,6\% $(\mathrm{n}=8)$ de 10 a 14 anos. Quanto à forma de ingresso na ESF do Jaboatão dos Guararapes, a maioria $(70,4 \% ; n=19)$ afirmou ter realizado concurso público.

Tabela 1. Caracterização demográfica e profissional da amostra. Jaboatão, PE, 2019.

\begin{tabular}{|c|c|c|}
\hline \multirow[t]{3}{*}{ Variável/Categoria } & \multicolumn{2}{|c|}{$\begin{array}{c}\text { Amostra } \\
\text { (frequência) }\end{array}$} \\
\hline & Absoluta & Frequência \\
\hline & $\mathbf{n}$ & $\%$ \\
\hline TOTAL & 27 & 100 \\
\hline \multicolumn{3}{|l|}{ Idade (em anos) } \\
\hline 28 a 38 & 18 & 66,7 \\
\hline 41 a 55 & 4 & 14,8 \\
\hline Não informado & 5 & 18,5 \\
\hline \multicolumn{3}{|l|}{ Sexo } \\
\hline Feminino & 19 & 70,4 \\
\hline Masculino & 3 & 11,1 \\
\hline Não informado & 5 & 18,5 \\
\hline \multicolumn{3}{|c|}{ Tempo de formado (em anos) } \\
\hline 4 a 6 & 5 & 18,5 \\
\hline 7 a 9 & 6 & 22,2 \\
\hline 10 a 14 & 5 & 18,5 \\
\hline 15 a 19 & 4 & 14,8 \\
\hline 24 a 30 & 2 & 7,4 \\
\hline Não informado & 5 & 18,5 \\
\hline \multicolumn{3}{|c|}{ Tempo de atuação na ESF (em anos) } \\
\hline Menos de 1 (um) ano ${ }^{1}$ & 1 & 3,7 \\
\hline 1 a 3 & 11 & 40,7 \\
\hline 4 a 8 & 3 & 11,1 \\
\hline 10 a 14 & 8 & 29,6 \\
\hline Não informado & 4 & 14,8 \\
\hline \multicolumn{3}{|c|}{ Forma de Ingresso na ESF } \\
\hline Concurso & 19 & 70,4 \\
\hline Seleção simplificada & 3 & 14,8 \\
\hline Outros & 1 & 3,7 \\
\hline Não informado & 4 & 11,1 \\
\hline
\end{tabular}

3 meses

Na Tabela 2, encontram-se os resultados relativos ao vínculo da eSB com a população adscrita à USF. Os CD participantes afirmaram conhecer o território $(70,4 \% ; n=19)$ e trabalharem integrados aos Agentes Comunitários de Saúde (ACS) (74,1\%; $n=20)$. Quando indagados sobre o estabelecimento de vínculo com a população adscrita $92,6 \%(n=25)$ afirmaram possuir vínculo. Em relação às famílias, 48,1\% $(\mathrm{n}=13)$ e 44,4\% $(\mathrm{n}=12)$ relataram possuir vínculo forte e intermediário, respectivamente. Em relação aos usuários da USF, 77,8\% $(\mathrm{n}=21)$ afirmaram estabelecer vínculo 


\section{$51 / /$}

forte. Relativo às organizações comunitárias, 59,3\% $(\mathrm{n}=16)$ estabelecem vínculo intermediário.

Um percentual de 55,6\% $(n=15)$ dos $C D$ relataram que a eSB não participa de ações organizadas pela comunidade. Dos 44,4\% (n=12) que afirmaram participar dessas ações, 47,1\% $(n=8)$ alegaram serem em associação de moradores, 35,3\% $(n=6)$ em igrejas/grupos religiosos e 17,6\% $(n=3)$ em escolas. Predominantemente, as ações realizadas pelos CD nesses espaços foram de característica preventiva $(81,0 \% ; \mathrm{n}=17)$

Tabela 2. Vínculo da eSB com a população adstrita à USF. Jaboatão, PE, 2019.

\begin{tabular}{|c|c|c|}
\hline \multirow{3}{*}{ Variável/Categoria } & \multicolumn{2}{|c|}{$\begin{array}{c}\text { Amostra } \\
\text { (frequência) }\end{array}$} \\
\hline & Absoluta & Frequência \\
\hline & $\mathbf{n}$ & $\%$ \\
\hline TOTAL & 27 & 100,0 \\
\hline \multicolumn{3}{|c|}{ A eSB conhece o território adscrito da USF } \\
\hline $\operatorname{Sim}$ & 19 & 70,4 \\
\hline Às Vezes & 8 & 29,6 \\
\hline Não & 0 & 0,0 \\
\hline \multicolumn{3}{|c|}{ A eSB trabalha integrada aos ACS } \\
\hline $\operatorname{Sim}$ & 20 & 74,1 \\
\hline Às Vezes & 7 & 25,9 \\
\hline Não & 0 & 0,0 \\
\hline \multicolumn{3}{|c|}{ A eSB estabelece vínculos com a população adscrita } \\
\hline $\operatorname{Sim}$ & 25 & 92,6 \\
\hline Às Vezes & 2 & 7,4 \\
\hline Não & 0 & 0,0 \\
\hline \multicolumn{3}{|c|}{ Tipo de vínculo com as famílias } \\
\hline Vínculo fraco & 2 & 7,4 \\
\hline Vínculo intermediário & 12 & 44,4 \\
\hline Vínculo forte & 13 & 48,1 \\
\hline \multicolumn{3}{|c|}{ Tipo de vínculo com os usuários } \\
\hline Vínculo fraco & 0 & 0,0 \\
\hline Vínculo intermediário & 6 & 22,2 \\
\hline Vínculo forte & 21 & 77,8 \\
\hline \multicolumn{3}{|c|}{ Tipo de vínculo com organizações comunitárias } \\
\hline Vínculo fraco & 6 & 22,2 \\
\hline Vínculo intermediário & 16 & 59,3 \\
\hline Vínculo forte & 5 & 18,5 \\
\hline \multicolumn{3}{|c|}{ Participação da eSB em ações organizadas pela comunidade } \\
\hline $\operatorname{Sim}$ & 4 & 14,8 \\
\hline Às Vezes & 8 & 29,6 \\
\hline Não & 15 & 55,6 \\
\hline Se sim/às vezes: & 12 & 44,4 \\
\hline \multicolumn{3}{|l|}{ - Local $^{1}$} \\
\hline Associação de moradores & 8 & 47,1 \\
\hline
\end{tabular}


Escolas municipais

Igrejas/grupos religiosos

- Característica da atividade

Ações educativas e preventivas (cárie/doença periodontal) ${ }^{2}$

Promotora de saúde 3
3

6

17,6

35,3

81,0

19,0

(1) Considerando que o mesmo pesquisado poderia citar mais de uma atividade.

(2) Ações: palestras, orientação de higiene bucal, escovação supervisionada e aplicação tópica de flúor.

(3) Ação: reunião entre profissionais e a comunidade, movimento à favor da USF e desenvolvimento de atividade física.

Os dados relativos à atuação da eSB nas práticas de VSB encontram-se na Tabela 3. Os resultados mostraram que $51,9 \%(\mathrm{n}=14)$ afirmaram às vezes realizar levantamentos epidemiológicos, sendo realizados semestralmente $(50,0 \%)$ e anualmente $(33,3 \%)$. Dos problemas avaliados, a cárie dentária foi a de maior frequência $(51,9 \%)$ dentre as respostas dos participantes, sendo os escolares (crianças e adolescentes) o grupo alvo mais avaliado pelos CD nestes levantamentos epidemiológicos (61,9\%). Quanto aos indicadores de autopercepção em saúde bucal, 70,4\% (n=19) alegaram não utilizá-los.

A maioria dos CD afirmam realizar busca ativa por problemas de saúde bucal ( $\operatorname{sim}=37,0 \%$; as vezes $=48,1 \%)$. A cárie dentária (32,7\%), as lesões orais $(20,4 \%)$ e o câncer bucal $(22,4 \%)$ foram os problemas de saúde bucal de maior frequência nas respostas dos participantes.

Os dados mostraram que a eSB realiza visitas domiciliares permanentes e sistemáticas $(\operatorname{sim}=59,3 \%$; as vezes $=37,0 \%)$ com o objetivo de garantir o acesso à saúde bucal aos usuários do território que se encontram acamados ou possuem dificuldade de locomoção até a USF (idosos, pacientes especiais, acidentados) (42,9\%). Os dentistas alegam realizar ações preventivas (19,0\%) de orientação de higiene bucal e de alimentação para os usuários em geral e para gestantes/puérperas, além do atendimento odontológico $(19,0 \%)$ onde prestam procedimentos clínicos viáveis para o momento e a marcação ao consultório. Também alegaram realizar nessas visitas a busca ativa de cárie, doenças periodontais e outras lesões orais não encontrados pelos ACS no território (19,0\%).

Os entrevistados afirmaram realizar ações de educação em saúde $(74,1 \%, n=20)$. As palestras $(37,7 \%)$, a demonstração de técnicas $(34,0 \%)$ e as atividades lúdicas $(18,9 \%)$ foram as metodologias de educação em saúde utilizadas com maior frequência nas respostas dos participantes sendo a higiene bucal $(24,1 \%)$ a temática mais frequente. 
Tabela 3. Atuação da eSB em práticas de VSB no Território. Jaboatão, PE, 2019.

Variável/Categoria $\quad \begin{gathered}\text { Amostra } \\ \text { (frequência) }\end{gathered}$

\begin{tabular}{|c|c|c|}
\hline & $\begin{array}{c}\text { Absoluta } \\
\text { n }\end{array}$ & $\begin{array}{c}\text { Frequência } \\
\%\end{array}$ \\
\hline TOTAL & 27 & 100,0 \\
\hline \multicolumn{3}{|c|}{ Realização de levantamentos epidemiológicos } \\
\hline Sim & 5 & 7,4 \\
\hline Às Vezes & 14 & 51,9 \\
\hline Não & 8 & 14,8 \\
\hline Se sim/às vezes, & 19 & $66,7 \%$ \\
\hline
\end{tabular}

\section{- Periodicidade ${ }^{1}$}

Anual $6 \quad 33,3$

Semestral/trimestral $9 \quad 50,0$

Mensal

2

11,1

Não informado

- Problemas avaliados ${ }^{1}$

Cárie dentária

\section{Variável/Categoria}

Amostra

(frequência)

Lesão de boca

Absoluta

n

Frequência

Doença periodontal

Outros

Não informado

\section{- Grupos alvos ${ }^{1}$}

Escolares (criança e adolescente)

Todas as faixas etárias

Adultos/idosos

Não informado

Utilização de indicadores de autopercepção em saúde bucal

Sim

Às Vezes

Não

Não informado 70,4

Realiza ações de busca ativa

Sim 25,9

Às Vezes 37,0

Não 48,1 14,8

Se sim/às vezes 85,1

- Busca ativa ${ }^{1}$

Cárie dentária (necessidade tratamento/dor/abscesso)

Doença periodontal

Câncer Bucal 
Necessidade de prótese

Usuários com dificuldade de autocuidado em saúde bucal

\section{Realiza visitas domiciliares}

Sim

Às Vezes

Não

Se sim/às vezes

- Finalidade das visitas domiciliares da eSB$^{1}$

Garantir acesso à saúde para pacientes acamados/dificil locomoção

Realizar ações preventivas

Atendimento odontológico

Busca ativa

Realiza ações de educação em saúde

Sim

Às Vezes

Não

\section{Se Sim/Às vezes}

- Metodologias de educação em saúde utilizadas ${ }^{1}$

Palestras

Demonstração de técnicas

Atividades lúdicas

Rodas de conversa/Troca de experiência

- Temáticas mais freqüentes ${ }^{1}$

Cárie Dentária

Câncer Bucal

Higiene Bucal

Doença periodontal

(1) Considerando que o mesmo pesquisado poderia citar mais de uma atividade.
6,1

8,2

59,3

37,0

3,7

96,3

26

42,9

19,0

19,0

19,0
74,1

22,2

3,7

1

37,7

34,0

18,9

$\begin{array}{ll}10 & 9,4\end{array}$

A Tabela 4 refere-se aos dados sobre a integração da eSB no planejamento das ações de saúde. Os dados afirmam que eSB realiza um-planejamento integrado com a eSF e o mapa inteligente é utilizado nas discussões de planejamento de ações $(51,9 \% ; n=14)$.

Os resultados mostraram que 62,9\% $(\mathrm{Sim} / \mathrm{n}=9$; As vezes $/ \mathrm{n}=8)$ dos $\mathrm{CD}$ integram as práticas de VSB com as de VS. A hipertensão arterial (24,2\%) e a diabetes $(24,2 \%)$ foram os problemas de saúde geral de maior frequência nas respostas dos participantes além de outros problemas alvo de vigilância da saúde $(21,2 \%)$.

Os CD alegaram realizar ações de educação em saúde no território ( $\operatorname{sim} / a ̀ s$ vezes=96,3\%;n=26) sendo a Alimentação saudável a temática mais comumente escolhida nessas ações e a seleção destas temáticas ter o envolvimento das equipes de saúde (eSB e eSF) e usuários $(38,5 \% ; n=10)$. 
Tabela 4. Integração da eSB com a eSF no planejamento das ações de saúde. Jaboatão, PE, 2019.

\begin{tabular}{|c|c|c|}
\hline \multirow[t]{3}{*}{ Variável/categoria } & \multicolumn{2}{|c|}{$\begin{array}{c}\text { Amostra } \\
\text { (frequência) }\end{array}$} \\
\hline & Absoluta & Frequência \\
\hline & $\mathbf{n}$ & $\%$ \\
\hline TOTAL & 27 & 100,0 \\
\hline \multicolumn{3}{|l|}{ Realiza planejamento integrado com a eSF } \\
\hline $\operatorname{Sim}$ & 23 & 85,2 \\
\hline Às Vezes & 4 & 14,8 \\
\hline Não & 0 & 0,0 \\
\hline \multicolumn{3}{|c|}{ O mapa de área é utilizado nas discussões de planejamento de ações } \\
\hline Sim & 14 & 51,9 \\
\hline Às Vezes & 8 & 29,6 \\
\hline Não & 5 & 18,5 \\
\hline \multicolumn{3}{|c|}{ Existem praticas integrada de VSB e VS no território } \\
\hline Sim & 9 & 33,3 \\
\hline Às Vezes & 8 & 29,6 \\
\hline Não & 9 & 33,3 \\
\hline Não informado & 1 & 3,7 \\
\hline Se sim/às vezes: & 17 & 62,9 \\
\hline \multicolumn{3}{|l|}{ - Em atividades de busca ativa para: ${ }^{1}$} \\
\hline Hipertensão arterial & 8 & 24,2 \\
\hline Diabetes & 8 & 24,2 \\
\hline Sífilis & 2 & 6,1 \\
\hline HIV & 2 & 6,1 \\
\hline Tuberculose & 3 & 9,1 \\
\hline Problemas psiquiátricos/ transtornos mentais & 3 & 9,1 \\
\hline Outros problemas alvo de Vigilância da Saúde ${ }^{2}$ & 7 & 21,2 \\
\hline Se sim/às vezes: ${ }^{3}$ & 26 & 96,3 \\
\hline \multicolumn{3}{|c|}{ - Temáticas mais frequentes em ações de educação em saúde ${ }^{1}$} \\
\hline Alimentação saudável & 12 & 22,2 \\
\hline Saúde sexual/DST & 3 & 5,6 \\
\hline Cuidados na gravidez/com recém-nascido & 5 & 9,3 \\
\hline Outras $^{4}$ & 8 & 14,8 \\
\hline \multicolumn{3}{|c|}{ - A seleção das temáticas das ações de educação em saúde envolve } \\
\hline Apenas a eSB & 5 & 19,2 \\
\hline Equipes de eSB e eSF & 8 & 30,8 \\
\hline \multirow[t]{3}{*}{ Variável/Categoria } & \multicolumn{2}{|c|}{$\begin{array}{c}\text { Amostra } \\
\text { (frequência) }\end{array}$} \\
\hline & Absoluta & Frequência \\
\hline & $\mathbf{n}$ & $\%$ \\
\hline eSB e usuários & 3 & 11,5 \\
\hline Equipes de eSB e eSF e usuários & 10 & 38,5 \\
\hline
\end{tabular}


(1) Considerando que o mesmo pesquisado poderia citar mais de uma atividade

(2) Outros problemas: hanseníase; hepatite; abandono/negligência; câncer de mama; deficiência nutricional.

(3) Amostra que respondeu sim/as vezes realizar ações de educação em saúde no território.

(4) Outras temáticas: higiene corporal; tabagismo; hipertensão; biossegurança; complicação em cirurgias; medicações; hábitos deletérios; doenças crônicas.

\section{DISCUSSÃO}

Com este estudo pode-se conhecer as práticas de VS desenvolvidas pelas eSB da ESF do município de Jaboatão dos Guararapes, numa perspectiva ampliada.

A caracterização dos participantes, respondentes $\mathrm{CD}$, quanto ao sexo, obteve resultados semelhantes a outros estudos ${ }^{14,15,16}$ realizados na APS que também identificaram um predomínio de CD do sexo feminino. Mesma situação vem sendo registrada para as demais profissões de saúde discutindo-se que esse processo de feminização da força de trabalho em saúde, deve-se em parte à crescente inclusão de mulheres nos cursos da área observada desde a década de 1980 e nos diferentes campos de trabalho da área. ${ }^{17}$

Ao considerar a idade e o tempo de formado, os resultados apontaram para um perfil de profissionais jovens com a maioria possuindo entre um a três anos de experiência de atuação na ESF. Resultados semelhantes foram encontrados em um estudo realizado por Casotti ${ }^{15}$ onde $44,4 \%$ da amostra de CD tinham até quatro anos de atuação na rede da APS. Diferentemente, um estudo ${ }^{16}$ identificou entre CD da ESF do Recife idades mais elevadas desses profissionais.

Em relação ao tipo de ingresso dos entrevistados deste estudo, a maioria afirmou ter ingressado na ESF de Jaboatão através de concurso publico. Esse achado converge com os resultados de um estudo realizado por Baldani, Ribeiro, Gonçalves e Ditterich ${ }^{18}$ que ao analisar a organização do processo de trabalho das eSB na ESF do estado do Paraná, identificaram que $90,0 \%$ dos CD haviam ingressado no serviço por concurso público. No entanto, em contraponto, foram reveladas fragilidades na forma de contratação que era estabelecida por meio de contratos temporários, prestações de serviço ou de indicações sem que houvesse um processo de seleção. ${ }^{19}$ Essas formas precárias de contratação ocasionam uma indesejada rotatividade de profissionais nas equipes da ESF, pois como forma de complementação salarial buscam outros serviços públicos ou privados, fato que dificulta a construção vínculos fortes entre os .profissionais na ESF com a comunidade.

Uma das diretrizes da APS para o trabalho na ESF destaca-se a territorialização e a adstrição de forma a permitir o planejamento, a programação descentralizada e o desenvolvimento de ações setoriais e intersetoriais com foco em um território específico. Além do que essas ações compõem o leque das atribuições comuns requeridas às eSF. ${ }^{8}$

Em relação a estes aspectos a maioria dos participantes afirmou conhecer o território da sua USF e trabalhar integrado aos ACS para obtenção de informações sobre as reais condições de vida e 
dos problemas de saúde bucal das famílias da área de atuação da equipe. No entanto, discordando desse achado, um estudo realizado no Distrito Federal constatou, um maior envolvimento das eSB investigadas com as atividades clínicas-assistenciais do que na atuação de maneira integrada em ações no território, junto às famílias e a comunidade..$^{20} \mathrm{E}$ outro estudo observou que práticas odontológicas no SUS se apresentam de forma desarticulada ao processo de organização dos demais serviços de saúde da ESF. ${ }^{12}$

Da mesma forma, a maioria considerou possuir vínculo com a população adscrita, sendo relatado existir um vínculo forte com as famílias e os usuários e um vínculo intermediário com as organizações comunitárias, no entanto, mais da metade dos CD relatou que a sua eSB não participa de ações organizadas pela comunidade. Os que participam restringem suas ações a atividades preventivas. Concordância foi obtida com outro estudo semelhante realizado em Parnamirim, Rio Grande do Norte, onde foi verificado que os CD participavam ocasionalmente de atividades na comunidade, denotando um vínculo comunitário fraco. ${ }^{19}$ Contudo, um estudo verificou uma atuação integrada e vinculada aos territórios das eSF e eSB investigadas, com a finalidade de realizar ações de natureza intersetorial. ${ }^{20}$ Em Recife, Pernambuco, foi observada uma integração razoável das eSB com a comunidade, porém com atuação junto a comunidade escolar. ${ }^{21}$

Nessa discussão, salienta-se que a dificuldade de estabelecimento de vínculo das equipes da ESF com a comunidade dos territórios da APS é uma questão reiteradamente abordada na literatura, sendo explicada as dificuldades dos profissionais em ampliar o escopo de suas ações para além da assistência em razão das tensões persistentes entre o modelo biomédico e o requerido para a $\mathrm{ESF}^{22}$

A observação e a análise permanente da situação de saúde da população prescindem de articulação intra e intersetorial por meio da integração de ações entre os setores de VS e da APS a fim de atender às necessidades de saúde da população de maneira integral com intervenções sobre os determinantes, os riscos e os agravos à saúde. , $^{2,5,8}$

Os resultados referentes às práticas de VSB no território mostraram lacunas, pois apenas uma minoria afirmou realizar levantamentos epidemiológicos, feitos anual ou semestralmente, focado na doença cárie e tendo como grupo alvo a comunidade escolar, apesar das orientações nacionais para a organização do processo de trabalho considerar as necessidades de saúde bucal de todos os ciclos de vida. ${ }^{23}$ Esses resultados foram concordantes com um estudo ${ }^{24}$ realizado em Natal, Rio Grande do Norte onde 70,0\% dos CDs não possuíam nenhum tipo de instrumento epidemiológico, sendo os únicos índices em uso relacionados apenas à cárie dentária e aos escolares. Constatandose negligenciamento quanto às recomendações nacionais sobre o uso do saber epidemiológico para subsidiar o planejamento das ações em saúde bucal. ${ }^{10,11,23}$

Ademais, cabem reflexões a respeito de ferramentas e meios que melhorem o acompanhamento epidemiológico da situação de saúde do território da APS pela eSB, subsidiando informações 
epidemiológicas para o planejamento de ações integradas das equipes da ESF. Além disso, é importante que haja uma maior aproximação das eSB com outros setores além da comunidade escolar para que, com isto, ocorra um trabalho interdisciplinar e intersetorial promovendo e prevenindo saúde de maneira integral.

Entretanto, a maioria dos entrevistados parece incluir de forma permanente e sistemática as visitas domiciliares e a busca ativa em seu processo de trabalho. Nas visitas prevaleceu entre os respondentes a intenção de garantir o acesso à saúde bucal dos indivíduos acamados ou com dificuldade de locomoção, E, na ação de busca ativa - para tratamento ou monitoramento da condição de saúde bucal -, o motivo prevalente foi a doença cárie, seguido do câncer bucal, das lesões de boca e doenças periodontais.

Em parte, esse resultado relaciona-se com os problemas de saúde bucal selecionados como alvo para a VSB das eSB da APS que estão apresentados na ficha odontológica individual do Sistema Integrado de Gestão da Saúde (E-SUS) da APS, devendo-se registrar os seguintes eventos: Abscesso dento alveolar, Alteração em tecidos moles, Dor de dente, Fendas ou Fissuras Lábio Palatais, Fluorose dentária moderada ou severa, Traumatismo dento alveolar. ${ }^{25}$

Tais achados reforçam os resultados já discutidos sobre o vínculo e demonstram um agir coerente com as atribuições requeridas as eSB e eSF que atuam na APS $^{12}$ sugerindo a produção de um cuidado integral em saúde bucal, na perspectiva da clínica ampliada, cuja organização consiste em um processo de trabalho que requer vínculo, responsabilização e resolutividade, para impactar diretamente no processo de produção em saúde e cuidado humanizado das pessoas. ${ }^{7,11}$

Diferentemente, outro estudo verificou em apenas uma parcela mínima de CD da APS a realização de visitas domiciliares, mas a busca ativa era direcionada para outros problemas como as lesões orais, como também observou-se neste estudo. ${ }^{24}$ Já em um estudo ecológico de base nacional sobre os processo de trabalho das eSB brasileiras mais da metade das eSB realizam campanhas para detecção de lesões suspeita de câncer de boca, entretanto, apenas 42,9\% delas encaminham os casos suspeitos ou diagnosticados, o que revela falhas na integralidade e coordenação do cuidado em saúde bucal. ${ }^{26}$

A intersetorialidade e o empoderamento comunitário são pontos-chave para a Promoção da Saúde pelo entendimento de que apenas um setor não consegue ser efetivo na resolução dos problemas de saúde que são complexos dada as múltiplas determinações sociais que estão envolvidas nos processos saúde-adoecimento.

Embora a maioria dos entrevistados tenha afirmado atuar em ações de promoção e educação em saúde com abordagem comunitária, predominaram práticas educativas conservadoras centradas em palestras e demonstração de técnicas sobre questões de higiene bucal e agravos em saúde e saúde bucal. Estas práticas conservadoras podem até provocar alguma mudança de comportamento 
individual na prevenção de doenças e agravos, no entanto não ocasionam mudanças significativas de comportamento organizacional, que necessitam de protagonismo e autonomia dos sujeitos envolvidos.

Sobre esse achado, obteve-se concordâncias com dois outros estudos ${ }^{27,28}$ que informaram uma predominância das práticas tradicionais de educação em saúde realizadas pelas eSF e pelas eSB apesar dos esforços para instituir um processo de trabalho na APS, a partir do reconhecimento da realidade social das famílias, com ênfase nas ações de promoção à saúde e no desenvolvimento de processos educacionais participativos. ${ }^{27}$ Divergindo desses resultados, em Pernambuco Pimentel, Albuquerque, Martelli, Souza e Acioli ${ }^{21}$ identificaram o interesse das eSB em promover com a comunidade debates sobre cidadania, assistência, funcionamento do SUS entre outros. Na Bahia, o estudo de Tavares, Costa, Falcão e Cristino ${ }^{29}$ não identificou a participação da comunidade na organização da oferta dos serviços prestados pelas eSB, revelando-se como fragilidade do Controle Social.

Solicita-se para a APS uma modalidade de trabalho ancorada no conceito de trabalho coletivo, realizado à luz dos pressupostos da VS e operacionalizado por meio da integração de saberes e práticas das diversas áreas de conhecimento no campo da saúde, no sentido de garantir uma visão mais abrangente sobre as necessidades de saúde e a integralidade da atenção. ${ }^{2}$

Sobre esses aspectos, a maioria dos entrevistados afirmou planejar ações de VS integradas aos demais membros da eSF e metade deles utiliza o mapa de área para subsidiar o planejamento de intervenções no território. Mas, menos da metade atua conjuntamente com as eSF, apenas em atividades de busca ativa e de educação em saúde. Concordâncias foram encontradas com um estudo que verificou uma atuação integrada e vinculada aos territórios das eSF e eSB investigadas, com a finalidade de realizar ações de natureza intersetorial. ${ }^{20} \mathrm{E}$ com outro estudo ${ }^{21}$ realizado em Recife, Pernambuco, quando observou-se existir uma integração razoável das eSB com a comunidade, com grande articulação e participação da comunidade escolar nas ações de saúde.

De um ângulo esses resultados sugerem engajamento das eSF em ações de saúde para além do escopo da saúde bucal. Por outro lado, podem estar revelando problemas de comunicação e relacionamento entre os membros das equipes podendo serem explicadas como já discutido pelas tensões entre o processo de trabalho em equipes colaborativas como indicado para a ABS e a persistência das práticas individualizadas do modelo assistencial em saúde anterior a reorganização das ações na APS do país. ${ }^{22}$ Segundo Fagundes, Thomaz, Queiroz, Rocha, Silva, Vissoci et al26 as práticas das eSB apresentam resultados contraditórios, sendo verificado avanços na direção da integralidade e persistências de práticas assistenciais restritas.

Sobre as limitações do estudo considera-se a baixa validade externa, por ser realizado em uma amostra de apenas três regionais de saúde, além do fato de não ter sido aplicado um questionário já validado. Por outro lado, têm-se como pontos fortes os cuidados metodológicos na construção 
(validação de face do instrumento) e na aplicação do instrumento de coleta de dados com a intenção de que os resultados expressassem a opinião dos respondentes, minimizando o viés do questionário em si e o viés de informação.

\section{CONCLUSÃO}

Os resultados deste estudo indicaram existir práticas de VSB no território que ainda possuem características restritas e dificuldades de estabelecimento de vínculos comunitários. Mas foram observados esforços de ampliação e atuação interprofissional como requerido para a APS do país.

A maioria das eSB não realizam levantamentos epidemiológicos para subsidiar o planejamento das ações de saúde bucal e quando efetuam concentram a atenção na doença cárie e na comunidade escolar. Percebeu-se resistência à quebra de práticas educativas tradicionais reforçando-se, o modelo conservador e hegemônico de atenção à saúde, que fragiliza a promoção da saúde e o empoderamento comunitário.

A integração de práticas de VS com os outros membros da eSF, foram observadas em ações de planejamento, educativas, de busca ativa e visitas domiciliares. Porém apresenta fragilidades referentes à exploração da participação da equipe em ações comunitárias.

\section{REFERÊNCIAS BIBLIOGRÁFICAS}

1. Pinto DS, Pereira BB, Limongi JE. Avaliação do conhecimento sobre Vigilância em Saúde entre os profissionais do Sistema Único de Saúde, Uberlândia, Minas Gerais. J. Health Biol Sci. [internet]. 2017. [Citado em: 27 fev. 2019]; 5(1):37-43. doi: http://dx.doi. org/10.12662/2317-3076jhbs.v5i1.1046.p37-43.2017.

2. Oliveira CM, Casanova AO. Vigilância da saúde no espaço de práticas da atenção básica. Ciência \& Saúde Coletiva [internet]. 2009. [Acesso em: 27 fev. 2019]; 14(3):929-936. Disponível em: https://www.scielosp.org/pdf/csc/2009.v14n3/929-936/pt

3. Teixeira CF, Paim JS, Vilasbôas AL. SUS, modelos assistenciais e vigilância da saúde. In: Rozenfeld S, organizadora. Fundamentos da vigilância sanitária. Rio de Janeiro: Editora FIOCRUZ; 2000, p. 49-60.

4. Faria LS, Bertolozzi MR. A vigilância na Atenção Básica à Saúde: perspectivas para o alcance da Vigilância à Saúde. Rev Esc Enferm USP [internet]. 2010 [citado em 27 fev. 2019]; 44(3):789-95. doi: https://doi.org/10.1590/S0080-62342010000300034.

5. BRASIL. Ministério da Saúde. Secretaria de Vigilância em Saúde. Secretaria de Atenção à Saúde. Diretrizes nacionais da vigilância em saúde. Brasília: Ministério da Saúde, 2010.

(Série B. Textos Básicos de Saúde) (Série Pactos pela Saúde 2006, 13). 
6. Monken M, Barcellos C. Vigilância em saúde e território utilizado: possibilidades teóricas e metodológicas. Cad. Saúde Pública [internet]. 2005 [citado em 27 fev. 2019]; 21(3):898-906. doi: http://dx.doi.org/10.1590/S0102-311X2005000300024.

7. Mattos GCM, Ferreira EF, Leite ICG, Greco RM. A inclusão da equipe de saúde bucal na Estratégia Saúde da Família: entraves, avanços e desafios. Ciência \& Saúde Coletiva [internet]. 2014 [citado em 27 fev. 2019]; 19(2):373-382. doi: http://dx.doi.org/10.1590/1413$\underline{81232014192.21652012 .}$

8. Goldstein RA, Barcellos C, Magalhães MAFM, Gracie R, Viacava F. A experiência de mapeamento participativo para a construção de uma alternativa cartográfica para a ESF. Ciência \& Saúde Coletiva [internet]. 2013 [citado em 27 fev. 2019]; 18(1):45-56. Disponível em: https:// www.scielosp.org/scielo.php?pid=S1413-81232013000100006\&script $=$ sci_arttext\&tlng $=\mathrm{en}$

9. Chaves SCL, Almeida AMFL, Rossi TRA, Santana AF, Barros SG, Santos CML. Política de Saúde Bucal no Brasil 2003-2014: cenário, propostas, ações e resultados. Ciência \& Saúde Coletiva [internet]. 2017 [citado em 27 fev. 2019]; 22(6):1791-1803. doi: http://dx.doi. org/10.1590/1413-81232017226.18782015.

10. Moysés SJ, Pucca Junior GA, Paludetto Junior M, Moura L. Avanços e desafios à Política de Vigilância à Saúde Bucal no Brasil. Rev Saúde Pública [internet]. 2013 [citado em 27 fev. 2019]; 47(Supl 3):161-7. doi: http://dx.doi.org/10.1590/S0034-8910.2013047004329.

11. Machado FCA, Souza GCA, Noro LRA. Indicators for teenager's oral health's surveillance. Ciência \& Saúde Coletiva [internet]. 2018 [citado em 27 fev. 2019]; 23(1):187-202. doi: https://doi.org/10.1590/1413-81232018231.20842015.

12. Sá CR, Kuhnen M, Santos IF, Arruda MP, Toassi RFC. PLANEJAMENTO EM SAÚDE BUCAL NA ATENÇÃO PRIMÁRIA À SAÚDE: DA TEORIA À PRÁTICA [internet]. 2015. [Acesso em: 27 fev. 2019]. Disponível em: https://periodicos.ufjf.br/index.php/aps/article/ view/15097

13. Secretaria Municipal de Saúde de Jaboatão dos Guararapes. Coordenação Municipal de Saúde Bucal. Saúde Bucal. 2017. 21 slides.

14. Garrido D, Huanca C, Oliveira A, Morita M, Haddad A. Feminisation of Dentistry in Brazil from the Perspective of a MOOC-Type Distance Course - A Short Report. J Int Soc Telemed eHealth [Internet]. 9Mar.2019 [cited 06Jun.2019]; 7:e6 (1-). Disponível em: https:// journals.ukzn.ac.za/index.php/JISfTeH/article/view/692

15. Casotti E, Jorge RC, Almeida PF, Santos AM. Atenção em saúde bucal em municípios sede de Regiões de Saúde do estado da Bahia. Diversitates Int J [internet]. 2017 [citado em 06 jun 
2019]; 09(1): 46-60. Disponível em: http://diversitates.uff.br/index.php/1diversitates-uff1/article/ view/172.

16. Silva CTC, Melo MMDC, Katz CRT, Carvalho EJA, Souza FB. Incorporação da técnica de restauração atraumática por equipes de saúde bucal da atenção básica à saúde do Recife/PE. Arq Odontol, Belo Horizonte [internet]. 2018 [citado em 06 jun 2019]; 54(06). doi: http://dx.doi. org/10.7308/aodontol/2018.54.e06

17. Matos IB; Toassi RFC; Oliveira MC. Profissões e ocupações de saúde eo processo de feminização: tendências e implicações. Athenea digital [internet]. 2013 [citado em 06 jun 2019]; 13(2):239-244. Disponível em: https://www.raco.cat/index.php/Athenea/article/view/291668

18. Baldani MH, Ribeiro AE, Gonçalves JRSN, Ditterich RG. Processo de trabalho em saúde bucal na atenção básica: desigualdades intermunicipais evidenciadas pelo PMAQ-AB. Saúde Debate, Rio de Janeiro [Internet]. 2018 [citado em 06 jun 2019]; 4(1):145-162. doi: http://dx.doi. org/10.1590/0103-11042018S110

19. Costa RM. O trabalho em equipe desenvolvido pelo cirurgião-dentista na Estratégia Saúde da Família: expectativas, desafios e precariedades [internet]. [S.1.]; 2012. [Acesso em: 3 jul. 2012]. Disponível em: https://rbmfc.org.br/rbmfc/article/view/434

20. Scherer CI, Scherer MDA, Chaves SCL, Menezes ELC. O trabalho em saúde bucal na Estratégia Saúde da Família: uma difícil integração?. Saúde debate, Rio de Janeiro [internet]. 2018 [citado em 06 jun 2019]; 42(02). doi: http://dx.doi.org/10.1590/0103-11042018s216

21. Pimentel FC, Albuquerque PC, Martelli PJL, Souza WV, Acioli RML. Caracterização do processo de trabalho das equipes de saúde bucal em municípios de Pernambuco, Brasil, segundo porte populacional: da articulação comunitária à organização do atendimento clínico. Cad. Saúde Pública, Rio de Janeiro [internet]. 2012 [citado em 06 jun 2019]; 28(Sup):S146-S157. Disponível em: https://www.scielosp.org/article/csp/2012.v28supp10/s146-s157/pt/

22. Esmeraldo GROV, Oliveira LC, Esmeraldo Filho CE, Queiroz DM. TENSÃO ENTRE O MODELO BIOMÉDICO E A ESTRATÉGIA SAÚDE DA FAMÍLIA: A VISÃO DOS TRABALHADORES DE SAÚDE. Rev. APS. [internet]. 2017 [citado em 06 jun 2019]; 20(1):98106. Disponível em: https://periodicos.ufjf.br/index.php/aps/article/view/15786

23. BRASIL. Ministério da Saúde. Secretaria de Atenção à Saúde. Departamento de Atenção Básica. Saúde Bucal. Brasília: Ministério da Saúde, 2008. 92 p. - (Série A. Normas e Manuais Técnicos) (Cadernos de Atenção Básica; 17) 
24. Almeida GCM, Ferreira MAF. Saúde bucal no contexto do Programa Saúde da Família: práticas de prevenção orientadas ao indivíduo e ao coletivo. Cad. Saúde Pública, Rio de Janeiro [internet]. 2008 [citado em 06 jun 2019]; 24(9):2131-2140. Disponível em: https://www.scielosp. org/scielo.php?script=sci_arttext\&pid=S0102-311X2008000900019

25. Brasil. E-SUS Sistema Integrado de Gestão da Saúde [homepage na internet]. Ficha de Atendimento Odontológico Individual.[acesso em 11 jun 2019]. Disponível em: http://www. rgesus.com.br/index.php/esus.html

26. Fagundes DM, Thomaz EBAF, Queiroz RCS, Rocha TAH, Silva NC, Vissoci JRN et al. Diálogos sobre o processo de trabalho em saúde bucal no Brasil: uma análise com base no PMAQ-AB. Cad. Saúde Pública [internet]. 2018 [citado em 11 jun 2019]; 34(9):383-391. doi: https://doi.org/10.1590/0102-311X00049817

27. Sityá D, Giacomini G, Sangioni L, Sendtko C, Unfer B. Análise de programas escolares de saúde bucal no Brasil. RFO [Internet]. 24abr.2015 [citado 12 jun 2019];19(3). Disponível em: http://seer.upf.br/index.php/rfo/article/view/3943

28. Mendes JDR, Freitas CASL, Dias MSA, Bezerra MM, Netto JJM, Fernandes DR. ANÁLISE DAS ATIVIDADES DE EDUCAÇÃO EM SAÚDE REALIZADAS PELAS EQUIPES DE SAÚDE BUCAL. Rev Bras Promoç Saúde, Fortaleza [internet]. 2017 [citado em 12 jun 2019]; 30(1): 13-21. doi: https://doi.org/ 10.5020/18061230.2017.p13

Tavares RP, Costa GC, Falcão MLM, Cristino PS. A organização do acesso aos serviços de saúde bucal na estratégia de saúde da família de um município da Bahia. Saúde em Debate [internet]. 2013 [citado em 12 jun 2019]; 37: 628-635. Disponível em: https://www.scielosp.org/article/sdeb/2013.v37n99/628-635/ pt/A ouvid

Artigo apresentado em outubro de 2019 Artigo aprovado em janeiro de 2020 Artigo publicado em maio 2020 